\title{
ÉVOLUTION DE LA RÉSISTIVITÉ DES LAMES MINCES DE NICKEL PHOSPHORE SOUS L'INFLUENCE DE LA TEMPÉRATURE
}

\author{
J. FLECHON, F. MACHIZAUD \\ Laboratoire de Physique de Dépôts Métalliques, Université Nancy I \\ et G. MARCHAL \\ Laboratoire d'Electronique et de Physique du Solide, Ensem, Nancy \\ (Reçu le 9 décembre 1971, révisé le 27 avril 1972)
}

\begin{abstract}
Résumé. - Nous voulons rendre compte dans le présent travail de l'importante décroissance de résistivité qui accompagne le recuit de l'alliage $\mathrm{Ni}-\mathrm{P}(10 \%$ de $\mathrm{P}$ en masse $)$, dans le domaine où cette substance demeure très imparfaitement cristallisée. Une analyse des gaz occlus, libërés pendant le chauffage, révèle la présence d'hydrogène. Toutefois la désorption de ce gaz ne saurait à elle seule produire une augmentation de $90 \%$ de la conductivité.

Une étude structurale aux rayons $\mathrm{X}$, au diffracteur d'électrons et une analyse thermique différentielle conduite sur l'alliage, nous amènent à conclure à l'existence dans la substance initiale de microcristaux de phosphore d'une part et de petits domaines constituant une phase amorphe d'autre part. Ces domaines sont formés par l'empilement plus ou moins désordonné de rubans plans hexagonaux parallèles.

La disparition des microcristaux de phosphore et l'évolution de la structure des domaines permettent d'expliquer le phénomène irréversible d'accroissement de conductivité observé avant $280^{\circ} \mathrm{C}$.

Abstract. - Our objectives in this work are to present the important decrease in resistivity which takes place during the tempering of the Ni-P alloy (10\% in mass), as far as this material remains imperfectly crystallysed. An analysis of the absorbed gas released during heating reveals the presence of hydrogen. However, the emission of hydrogen could not produce an increase of $90 \%$ in the conductivity.

A structural study, with the help of $\mathrm{X}$ rays and electron diffraction, as well as a differential thermal analysis made upon the alloy, leads us to the conclusion that there exists in the initial substance phosphorus microcrystals on the one hand, and small regions forming an amorphous phase on the other hand. Those regions are made up of flat hexagonal parallel tapes piled in a way which is more or less irregular.

The disappearance of phosphorus microcrystals and the evolution of the structure of those regions allow us to explain the irreversible phenomenon of increasing conductivity observable under $280^{\circ} \mathrm{C}$.
\end{abstract}

L'oxydo-réduction de l'acétate de nickel par l'hypophosphite de sodium permet d'obtenir en phase liquide dans des conditions convenables [1] des lames minces de nickel phosphore d'épaisseur variant de quelques centaines à quelques milliers d'angströms. L'intérêt de ces lames réside dans le fait que la matrice est quasi amorphe ou très imparfaitement cristallisée. La présence du métalloïde libéré au cours de la réaction n'est pas étrangère à cet état.

Un apport contrôlé d'énergie thermique étend progressivement l'ordre local à un ordre à grande distance. Au cours de cette transformation conduite sous vide classique de $10^{-6}$ torr, la résistivité électrique diminue dans des proportions variant avec la température de traitement, mais pouvant ramener la résistance à $3 \%$ de sa valeur initiale. Les températures de traitement passent de l'ambiante à $500^{\circ} \mathrm{C}$. L'inter- prétation des phénomènes observés conduit aux questions suivantes:

$1^{\circ}$ Est-il possible d'associer un mécanisme déterminé à une diminution aussi importante de la résistivité ?

$2^{\circ}$ Comment prouver lá réalité du processus avancé ?

I. Mécanisme possible. - Si plusieurs types de transformations sont à l'origine de l'augmentation de la conductivité électrique des lames, ils seront isolables par un chauffage programmé sous vide avec mesure en continu de la résistance électrique de l'échantillon étudié [2]. En effet, d'après Damask et Dienes [3], le chauffage à vitesse constante permet de mettre en évidence les températures de recuit caractérisant une étape donnée de l'évolution de la matrice. 
Si $n$ est le nombre d'événements élémentaires liés à une transformation de la substance,

$$
\frac{\mathrm{d} n}{\mathrm{~d} t}=-F(n) K=-F(n) \frac{K o \mathrm{e}^{-E}}{k T},
$$

les lettres ayant une signification classique.

Si la résistivité $\rho$ est une fonction de $n$, au voisinage d'une température de transformation $T_{\mathrm{c}}$, ( $-\mathrm{d} \rho / \mathrm{d} t$ ) faible en dessous de $T_{\mathrm{c}}$ subira une brusque augmentation au passage à cette température, puis diminuera ensuite, le domaine de variation permettant de définir l'intervalle de température caractérisant le phénomène.

L'évolution de la résistivité électrique de lames minces de Ni-P ( $\simeq 1500 \AA, \simeq 10 \%$ de P en masse) obtenues à $25{ }^{\circ} \mathrm{C}$ sur verre plan et chauffées sous vide en «montée linéaire » est donnée par la figure 1. La vitesse de chauffage est de $0,2 \% / \mathrm{mn}$.

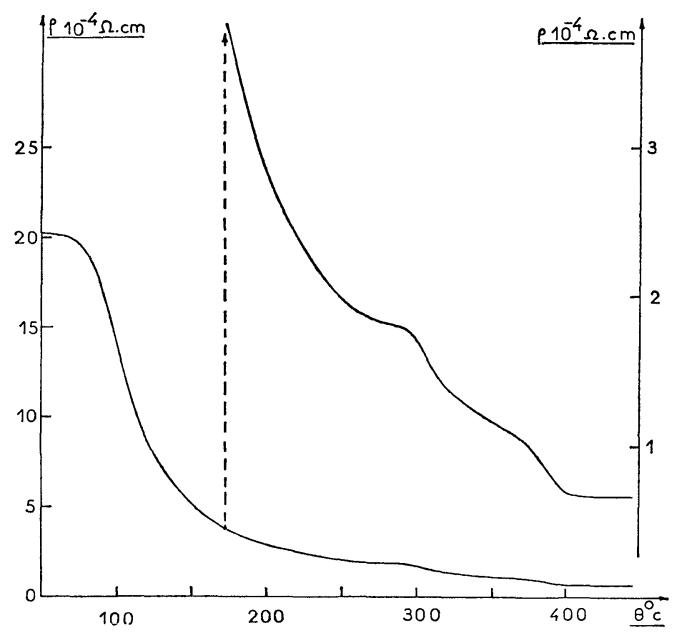

Fig. 1. - Résistivité électrique de l'alliage Ni-P en fonction de la température de recuit («montée linéaire ») : lames de $1500 \AA$ renfermant $10 \%$ de $\mathrm{P}$ en masse.

Trois domaines apparaissent clairement :

De $50^{\circ} \mathrm{C}$ à $280^{\circ} \mathrm{C}$ avec tangente inflexionnelle à $100{ }^{\circ} \mathrm{C}$ : domaine 1.

De $280^{\circ} \mathrm{C}$ à $350^{\circ} \mathrm{C}$ avec tangente inflexionnelle $\mathrm{a}^{\mathrm{w}} 300^{\circ} \mathrm{C}$ : domaine 2.

De $350^{\circ} \mathrm{C}$ à $430^{\circ} \mathrm{C}$ avec tangente inflexionnelle à $385^{\circ} \mathrm{C}$ : domaine 3 .

Ces trois régions sont exactement retrouvées par la mesure des coefficients de température $K_{\theta}=1 / \rho \mathrm{d} \rho / \mathrm{d} \theta . K_{\theta}$ qui décroît avec l'augmentation du taux de phosphore ne dépasse pas $400 \times 10^{-6}$ dans le domaine 1 pour des lames comportant $5 \%$ à $6 \%$ de phosphore.

Une étude structurale aux rayons $X$ [2] [4] révèle la présence de bandes de diffraction caractérisant dans le' domaine 1 la présence de substances très imparfaitement organisées.

L'échantillon soumis au rayonnement $\mathrm{X}$ est une poudre de même composition que les lames, préparée en quantité suffisante et bien homogénéisée afin de permettre par fractionnement des mesures sur un nombre important d'échantillons statistiquement identiques. Elle est stabilisée par recuits sous vide classique successivement à $50^{\circ} \mathrm{C}, 150^{\circ} \mathrm{C}, 250^{\circ} \mathrm{C}$, la durée de stabilisation étant définie par une étude préalable de la variation isotherme de la conductıvité électrique [15]. En effet, l'échantillon très imparfaitement cristallisé est en quasi-équilibre [16]. Sa structure évolue sous l'influence d'un apport contrôlé d'énergie thermique après passage par un état transitoire ou activé vers un état caractérisé par une énergie libre $G_{1}$ inférieure à l'énergie $G_{0}$ initiale $G_{0}-G_{1}=\Delta G$ définissant la force motrice de la réaction.

Après chaque recuit, les échantillons sont ramenés à la température ambiante pour en obtenir le spectre de rayons $\mathrm{X}$ définissant un stade déterminé dans l'évolution structurale du matériau.

Le diffractomètre employé (CGR théta 60) est muni d'un montage pas à pas au $1 / 10^{\mathrm{e}}$ de degré avec compteur complété par un discriminateur d'impulsions. L'ensemble de comptage a été utilisé en présélection du nombre de coups afin de travailler à précision constante. Le montage classique par réflexion permet d'effectuer le comptage jusqu'à la valeur $\theta=45^{\circ}$, limite imposée par la rotule de la chambre à vide utilisée. Les mêmes échantillons sont étudiés successivement avec deux rayonnements monochromatiques : Mo $K_{\alpha}$ et $\mathrm{Cu} K_{\alpha}$ ceci afin de connaître avec plus de précision les intensités aux faibles valeurs de $\theta$.

Après corrections des intensités expérimentales (fluorescence, polarisation, diffusion Compton, raccordement des diagrammes $\mathrm{Mo} K_{\alpha}$ et $\mathrm{Cu} K_{\alpha}$, mise à l'échelle absolue avec normalisation suivant la méthode d'Averbach, Strong et Kaplov [17]), et transformation de Fourier de la fonction d'interférences (Fig. 2),

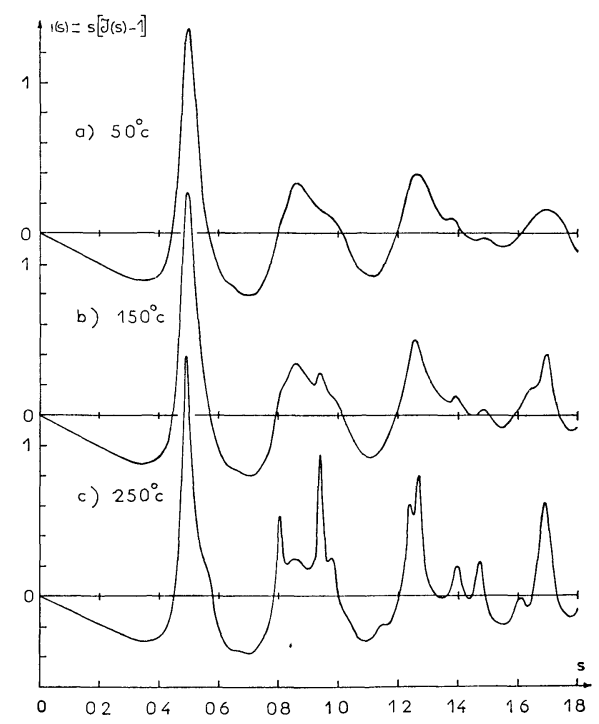

FIG. 2. - Fonctions d'interférences en fonction de $s=\frac{2 \sin \theta}{\lambda}$ : alliage Ni-P $(10,5 \%)$ recuit à $50^{\circ} \mathrm{C}, 150^{\circ} \mathrm{C}, 250^{\circ} \mathrm{C}$. 
on aboutit à la fonction de répartition

$$
p(r)=r(P(r)-1)
$$

(Fig. 3). L'examen de ces courbes à $50^{\circ} \mathrm{C}$ et $150^{\circ} \mathrm{C}$ ne semble pas révéler une évolution dans l'ordre local susceptible d'expliquer à elle seule l'importante chute de la résistivité observée dans le domaine 1 (Fig. 1).

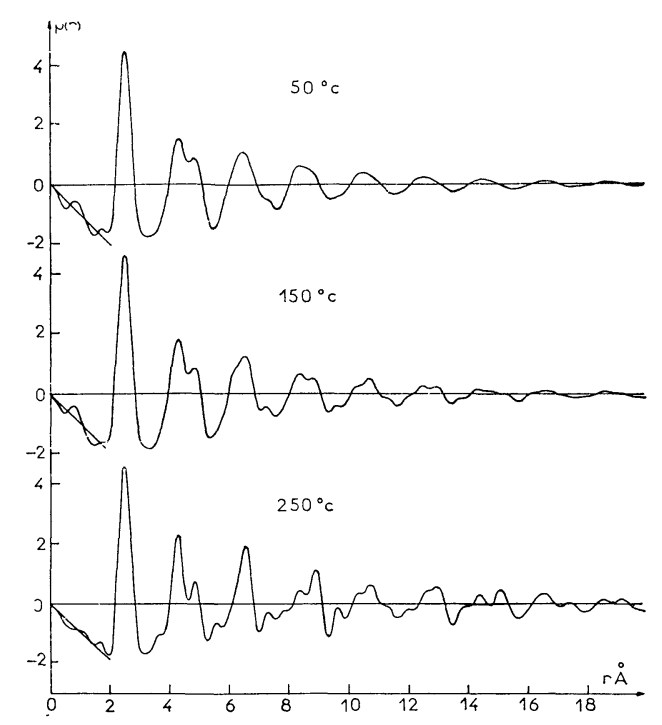

FIG. 3. - Fonctions de répartition de l'alliage Ni-P (10,5\%) recuit à $50^{\circ} \mathrm{C}, 150^{\circ} \mathrm{C}, 250^{\circ} \mathrm{C}$.

La génération des lames se produisant en phase liquide dans un milieu saturé d'hydrogène, il convenait de s'assurer si du gaz physisorbé ne modifiait pas de façon importante les propriétés de transport des lames.

II. Mise en évidence de la présence d'hydrogène dans les lames minces. - Un échantillon ayant une masse comprise entre 1 et $5 \mathrm{mg}$, la quantité de gaz occlus est faible et l'extraction suivie de caractérisation ne se révèle possible que par l'emploi d'une méthode spectrométrique.

Qualitativement, il est aisé de se rendre compte, par simple chauffage et lecture du vide à la jauge, qu'un dégazage accompagne l'apport d'énergie thermique.

Des essais comparatifs avec support vierge puis recouvert de couche métallique sont significatifs mais sans grand intérêt.

a) Dispositif expérimental (Fig. 4). - 1) Principe de l'appareil. - L'analyseur de gaz utilisé est un spectromètre de masse du type à résonance d'ions (omégatron), de dimensions très réduites, dont le principe est analogue à celui du cyclotron.

Dans une enceinte où règne un vide de $5 \times 10^{-7}$ torr, sont appliqués orthogonalement un champ magnétique constant $\mathbf{B}$ et un champ électrique alternatif $\mathbf{E}$ de pulsation $\omega$. Un faisceau d'électrons, très fin, paral-

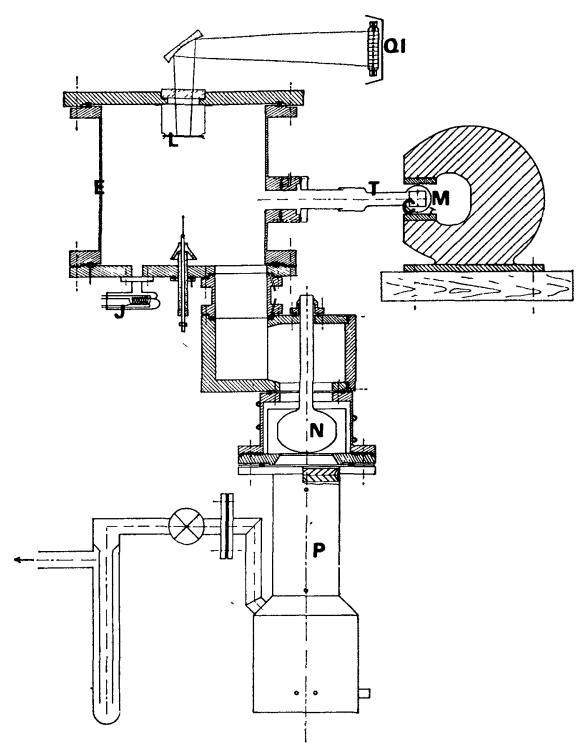

FIG. 4. - Dispositif expérimental, analyseur de gaz.

lèle à la direction du champ magnétique, ionise les molécules de gaz présentes dans l'enceinte et sous l'action des champs $\mathbf{E}$ et $\mathbf{B}$, les ions formés décrivent, autour du faisceau d'électrons où ils ont pris naissance, des trajectoires spirales dans un plan perpendiculaire à $\mathbf{B}$ [14].

Parmi ces ions, seules les particules de charge $e$ et de masse $m$ dont la fréquence cyclotron $\omega=e B / m$ est égale à la fréquence du champ électrique appliqué, décrivent des spirales dont les rayons vecteurs augmentent indéfiniment et peuvent ainsi atteindre un collecteur $\mathrm{C}$ situé à une distance déterminée du faisceau d'électrons. Ces ions, dits résonnants, abandonnent leurs charges sur le collecteur. Ainsi la mesure $\mathrm{du}$ courant ionique recueilli sur $\mathrm{C}$ renseigne sur la pression partielle des particules de masse $m$ présentes dans l'échantillon de gaz.

2) Circuits électriques. - Le champ électrique alternatif $\mathbf{E}$ est fourni par un wobulateur dont la fréquence peut varier de façon continue entre $50 \mathrm{kHz}$ et $5 \mathrm{MHz}$. Cet appareil est piloté par un générateur TBF dont la fréquence de balayage est réglée entre $10^{-3}$ et $5 \times 10^{-3} \mathrm{C} / \mathrm{s}$. Ce balayage est également appliqué à l'entrée $\mathrm{X}$ d'un enregistreur $\mathrm{X}-\mathrm{Y}$ dont la voie $\mathrm{Y}$ reçoit le courant d'ions après amplification par un électromètre à condensateur vibrant Cary. La constante de temps de cet électromètre détermine la durée d'enregistrement d'un spectre complet qui est toujours de l'ordre de 10 minutes. Nous avons essayé de diminuer ce temps en limitant le domaine de masse étudié aux masses 1 et 2 et en utilisant un balayage symétrique en dents de scie.

3) Dispositif d'étude. - L'examen de phénomène évoluant rapidement exclut l'utilisation des méthodes d'analyse microchimique qui nécessitent généralement des mesures volumétriques, longues et délicates où interviennent la température et la pression. Par ail- 
leurs, elles exigent des quantités de gaz relativement importantes. Les faibles masses de gaz libérés durant l'échauffement des lames minces, dont le poids n'excède pas dix milligrammes, peuvent représenter seulement quelques ppm ce que l'analyseur de gaz résiducl peut atteindre facilement. Il est d'autre part primordial de s'assurer que les gaz libérés durant l'échauffement de l'échantillon proviennent uniquement de celui-ci. D'où la nécessité de produire cet échauffement par un dispositif «propre», éliminant ainsi tout chauffage indirect à partir de filaments chauds qui sont des sources d'hydrogène (filament de W, C...) et de diminuer le rayonnement de cet échantillon vers les parois de l'enceinte en l'éloignant de celles-ci.

Ces impératifs nous ont conduits à réaliser le dispositif expérimental représenté figure 4. L'enceinte $\mathrm{E}$ est constituée d'un cylindre en acier inoxydable de diamètre égal à sa hauteur soit $300 \mathrm{~mm}$. Elle est reliée au spectromètre de masse $M$ par une tubulure $\mathrm{T}$ de conductance $11 \mathrm{l} / \mathrm{s}$ ' et à une pompe à diffusion d'huile $\mathbf{P}$ bafflée surmontée d'un piège à azote liquide $\mathrm{N}$. La pression totale est mesurée par une jauge de BayartAlpert $\mathbf{J}$ de conductance $8 \mathrm{l} / \mathrm{s}$. L'enceinte est fermée par une platine supérieure qui supporte les échantillons (2 lames $\mathrm{L}$ de pyrex $25 \times 75 \mathrm{~mm}$ recouvertes sur chaque face d'un dépôt métallique) suspendus par 4 fils de tungstène afin de diminuer les pertes thermiques par conduction.

Notons que les analyses ne seront quantitativement valables que si le spectromètre a un résiduel propre minimum : ceci impose que l'appareil soit de petites dimensions, que les effets de surface soient aussi réduits que possible et qu'il puisse être énergiquement dégazé.

Ces conditions ne peuvent être complètement satisfaites ici puisque l'étude des dégazages à une température inférieure à $200^{\circ} \mathrm{C}$ élimine tout dégazage préalable de l'enceinte du spectromètre. Nous avons donc surmonté partiellement ces difficultés en diminuant les dégazages parasites (augmentation des dimensions de l'enceinte et chauffage propre de l'échantillon).

Durant des essais préliminaires, les échantillons étaient chauffés par conduction à l'aide d'une plaque en duralumin dans laquelle était fixée une résistance chauffante en nickel-chrome. A l'aide de ce montage, la température des couches pouvait être portée à $400^{\circ} \mathrm{C}$, mais les spectres des gaz émis durant le chauffage ne permettaient pas de séparer clairement les gaz libérés par l'élément chauffant de ceux provenant des échantillons.

Nous avons résolu cette difficulté en faisant appel à un chauffage par rayonnement infra-rouge issu d'une lampe quartz-iode de $1000 \mathrm{~W}$ QI.

Le faisceau énergétique focalisé par un miroir concave pénètre dans l'enceinte par un hublot en pyrex (Fig. 4). La température maximale des couches, mesurée à l'aide d'un thermocouple collé à la pâte 'à l'argent sur la face inférieure des couches ne dépasse pas $180^{\circ} \mathrm{C}$. b) RÉsultats. - Une première série de mesures nous permet de définir, l'atmosphère résiduelle de l'enceinte. La figure 5 montre en échelle semi-logarithmique le spectre de masse des gaz qu'elle renferme. Les courants d'ions sont exprimés en ampères; la pression totale mesurée par une jauge Bayart Alpert est de $5 \times 10^{-7}$ torr.

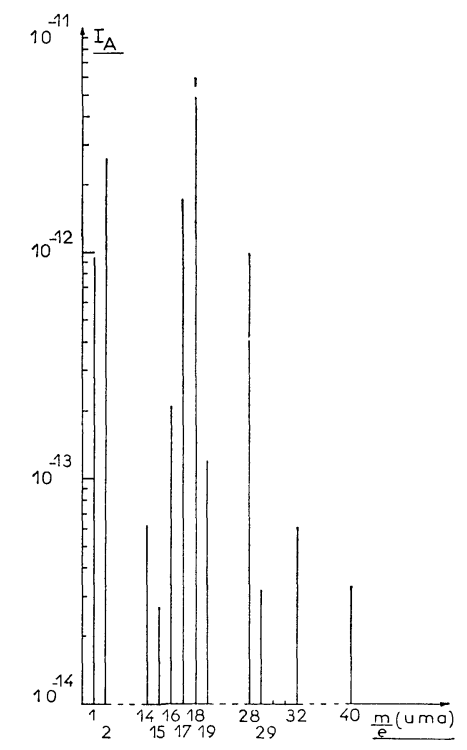

Fig. 5. - Spectre de masse des gaz de l'atmosphère résiduelle.

Les masses prépondérantes sont 1, 2, hydrogène atomique et moléculaire ( $28 \%$ de la pression totale), 28 , azote et oxyde de carbone $(8 \%$ de la pression totale) et 17,18 , vapeur d'eau ( $60 \%$ de la pression totale).

L'oxygène, l'azote, la vapeur d'eau présents dans l'enceinte non dégazée ne peuvent être éliminés, la présence de l'échantillon rendant difficile dans le montage adopté un étuvage préliminaire.

Des essais comparatifs nous ont permis de suivre l'évolution des pics en chauffant des supports d'abord seuls puis recouverts des lames minces métalliques. Dans le premier cas, les variations des amplitudes des pics suivent celles de la pression totale. Dans le second, la pression partielle d'hydrogène augmente d'environ $300 \%$, alors que la pression totale correspondante varie de $130 \%$ (Fig. 6). Ainsi le dégazage des couches minces sous l'action de la chaleur pourrait expliquer l'importante augmentation de la conductivité.

c) Critiques. - La méthode et l'appareillage que nous avons retenus appellent les critiques suivantes :

Il serait souhaitable d'utiliser un spectromètre de masse à temps de réponse beaucoup plus court.

En effet, les courants d'ions sont mesurés à l'aide d'un électromètre à condensateur vibrant Cary, dont le temps de réponse nous contraint d'effectuer un spectre en plusieurs minutes. D'autre part, le spectro- 


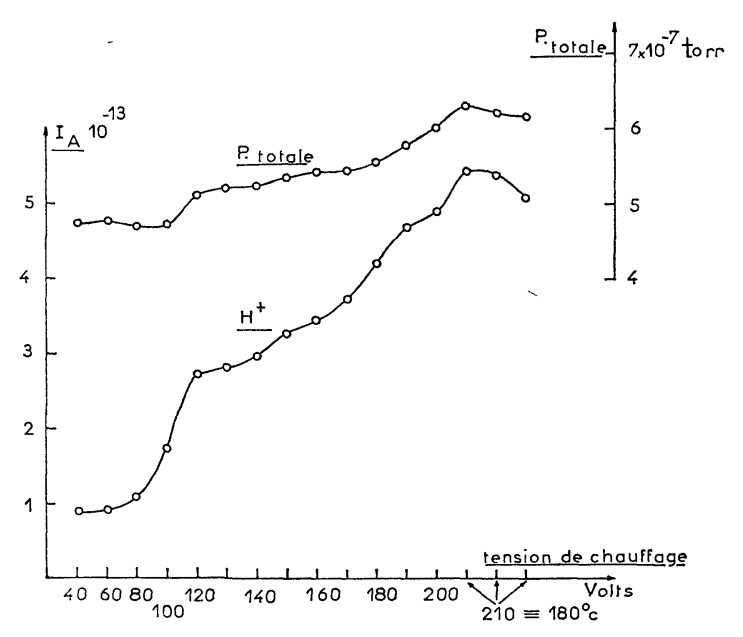

Fig. 6. - Pression partielle d'hydrogène (courants d'ions en ampères) et pression totale en torr en fonction de la tension de chauffage.

mètre utilisé possède une sensibilité différente selon le domaine de masse exploré.

Il a donc fallu régler la sensibilité à son maximum pour les masses 1 et 2 et effectuer un balayage en fréquences correspondant à ces deux masses. Il serait naturellement souhaitable d'obtenir le spectre complet à chaque instant et d'étendre l'expérience jusque vers $500^{\circ} \mathrm{C}$.

Il est cependant hors de doute que la variation de pression partielle d'hydrogène au cours du chauffage accompagne l'augmentation de la conductivité. Le problème est de savoir si l'importante diminution que l'on observe sur les résistances dépend du départ d'hydrogène. D'après Suhrmann [5], l'absorption d'hydrogène sur des films de nickel déposés par évaporation thermique sur substrats de verre ou de quartz à $90 \mathrm{~K}$ provoque une augmentation de la conductivité lorsque la matrice est cristallisée, une diminution lorsqu'elle est amorphe. Nous avons vu que seul règne un ordre local dans les films de Ni-P (Fig. 3), ce qui confirmerait nos observations.

Par ailleurs, Míostovetch [6] signale que l'absorption d'hydrogène par des films métalliques augmente leur résistivité lorsque le coefficient de température des résistances est positif, ce qui est le cas des lames de Ni-P.

Cependant, Suhrmann [5] signale que la variation observée n'excède pas $2 \%$ dans les meilleures conditions. Il convient donc de chercher une explication complémentaire d'une variation aussi importante de la conductivité entre $50^{\circ} \mathrm{C}$ et $150^{\circ} \mathrm{C}$.

III. Analyse thermique différentielle. - Afin d'éclairer le processus indiqué, une analyse thermique différentielle a été conduite par l'emploi simultané de deux thermocouples Chromel Alumel, montés en opposition sur un enregistreur Meci à point médian sensible à $2 \mu \mathrm{V}$ et plongés séparément dans deux échantillons pulvérulents obtenus dans des conditions iden- tiques à celles des dépôts en lames. L'un des échantillons a été recuit sous vide jusqu'à $500^{\circ} \mathrm{C}$ afin de servir de témoin, l'autre, simplement séché, fait l'objet de l'analyse thermique différentielle. La vitesse de chauffage était de $2 \% \mathrm{mn}$. Ce dispositif utilisé par A. W. Goldenstein [7] et J. P. Randin [8] se révèle d'un emploi commode dans l'étude présente.

Les résultats sont indiqués sur la figure 7 .

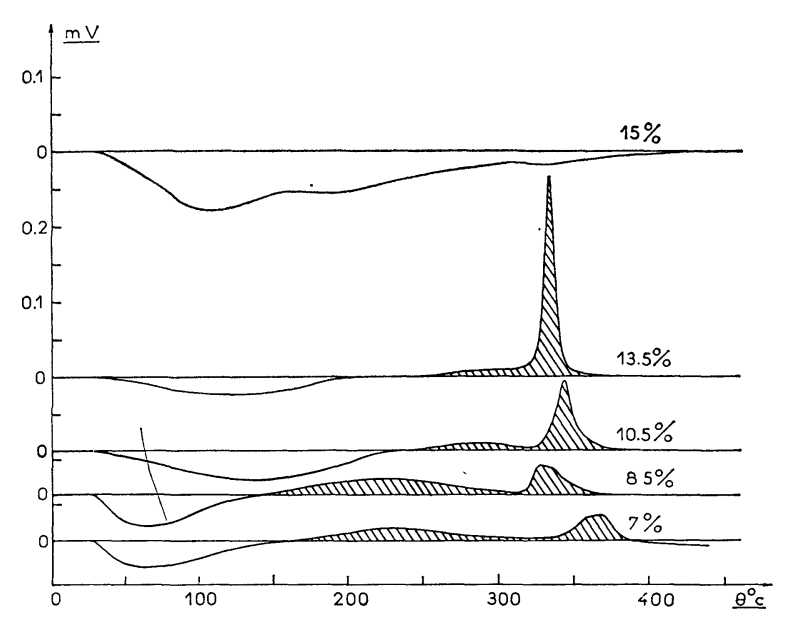

FIG. 7. - Analyse thermique différentielle pour les alliages Ni-P $7 \%, 8,5 \%, 10,5 \%, 13,5 \%, 15 \%$.

Quel que soit le taux en métalloïde, jusqu'à $200^{\circ} \mathrm{C}$ se produit un phénomène endothermique qui pourrait être la libération de gaz occlus. En particulier à $15 \%$ de $\mathbf{P}$ (composition stœchiométrique de $\mathrm{Ni}_{3} \mathrm{P}$ ), de $50^{\circ} \mathrm{C}$ à $450^{\circ} \mathrm{C}$ seule une transformation endothemique se produit. Mais pour toutes les substances moins riches en métalloïde, apparaît à partir de $300^{\circ} \mathrm{C}$ un pic exothermique d'autant plus important qu'augmente la quantité de phosphore présent dans le dépôt et correspondant à la formation du phosphure $\mathrm{Ni}_{3} \mathrm{P}$.

Cette étude jointe à celle aux rayons $\mathrm{X}$ permet de prouver que le phosphure de nickel $\mathrm{Ni}_{3} \mathrm{P}$ n'est pas présent dans les dépôts non recuits dont le taux en phosphore n'atteint pas $15 \%$; en poids.

Cette analyse nous permet d'affirmer, ainsi que l'a montré Flechon [9], que le phosphore n'est pas lié au nickel d'une manière aussi solide que dans $\mathrm{Ni}_{3} \mathrm{P}$. Il pourrait exister également à l'état de phosphore libre microcristallisé : en effet, les raies les plus intenses du métalloïde (phosphore noir) (002), (012), (004), (111), sont visibles sur les microdiffractions électroniques et les diagrammes $\mathrm{X}$. Ces raies disparaissent vers $150^{\circ} \mathrm{C}$.

Dans les alliages amorphes de GeAuSi, Turnbull [10] a pu observer une diminution de la conductivité par. formation de petits cristallites; ainsi la présence initiale de microcristaux de phosphore qui sont une entrave à la propagation des ondes électroniquies dans l'alliage Ni-P pourrait contribuer à sa forte résistivité électrique de même que leur disparition, suivie d'une 
diffusion du phosphore libre, à une croissance de la conductivité. Ce résultat est en accord avec les travaux de Viard [11] qui observe dans l'état homogène (alliage amorphe) une augmentation avec la température du nombre $\mathbf{n}$ d'électrons de conduction par atome : il considère, comme Maeda [12], Albert et Kovac [13] que le matériau est une solution saturée de phosphore dans le nickel ; les atomes de phosphore libre apparus après destruction des microcristaux participant à la conductivité, $n$ croît avec le taux du soluté.

IV. Interprétation des fonctions de répartition $p(r)$ : (Fig. 3). - L'ensemble de la fonction $p(r)$ de l'alliage recuit à $50^{\circ} \mathrm{C}$ permet d'envisager un arrangement hexagonal plan préfigurant les plans (111) de la structure cubique à faces centrées du nickel. En effet, nous n'observons aucun pic autre que ceux de l'ensemble des distances interatomiques d'un tel arrangement: notamment, absence des distances entre voisins de deux couches successives à $3,54 \AA$ et $5,59 \AA$ qui caractérisent un empilement compact.

La substance de départ serait donc formée d'un empilement de petits éléments plans parallèles, cristallisés dans deux dimensions, mais non ordonnés entre eux.

Cette conclusion est confirmée par la forme de la fonction d'interférences (Fig. 2a) composée de bandes de diffraction provenant des réseaux hexagonaux plan auxquelles viennent s'ajouter les réflexions de Bragg produites par les empilements

\begin{tabular}{|c|c|c|c|}
\hline \multirow{3}{*}{$\begin{array}{c}\text { Bandes } \\
\overline{(10)}\end{array}$} & \multirow{2}{*}{$\begin{array}{c}\text { Réflexions } \\
-\end{array}$} & \multicolumn{2}{|c|}{$s=\frac{2 \sin \theta}{\lambda}$ calculé } \\
\hline & & & - \\
\hline & - & 0,46 & nromion \\
\hline - - & $(002)$ & 0,49 & premier anneau \\
\hline (11) & - & 0,80 & \\
\hline (20) & - & 0,92 & deuxième anneau \\
\hline 一 & $(004)$ & 0,98 & \\
\hline (21) & - & 1,22 & \\
\hline (30) & - & 1,39 & troisième anneau \\
\hline- & (006) & $1,47)$ & \\
\hline (22) & - & 1,60 & ne anneau \\
\hline (31) & - & 1,66 & me anneau \\
\hline
\end{tabular}

Il est à noter une anomalie des intensités relatıves des pics de la fonction $p(r)$ de la rangée [10] : le pic à $5 \AA$ est trop faible par rapport à celui à $2,5 \AA$ et le pic à $7,5 \AA$ est à peine visible ; ce qui définit une distance de cohérence de l'ordre de 7,5 $\AA$ dans la direction [10]. Par contre, nous observons une décroissance plus lente des pics de la rangée [11] à 4,33 $\AA$, $8,66 \AA$ et $12,99 \AA$. Cette remarque nous conduit à imaginer des éléments plans ayant la forme de rubans allongés dans la direction [1]] : la distance de cohérence étant de l'ordre de 7,5 $\AA$ dans la direction [10] et voisine de $13 \AA$ dans la direction [11̄]

Si dans le plan d'un ruban la plus grande distance de cohérence est de $13 \AA$, notons cependant que la fonction $p(r)$ présente encore des oscillations jusqu'aux environs de $20 \AA \AA$. Or, pour des valeurs de $r$ supérieures à $8 \AA$ les oscillations présentent une périodicité de l'ordre de $2 \AA$ qui concorde avec celle de la succession des couches hexagonales dans la direction [002]. Ceci nous définit dans cette direction une distance de cohérence voisine de $20 \AA$.

Dans l'alliage initial, des rubans plans de petites dimensions $(\simeq 10 \AA)$, empilés sur une épaisseur d'une vingtaine d'angströms, définissent des domaines indépendants à l'intérieur desquels les couches parallèles peuvent avoir subi des translations ou des rotations dans leur plan. Une telle structure contribue certainement à la forte résistivité du matériau.

Après recuit de l'alliage à $150^{\circ} \mathrm{C}$ puis $250^{\circ} \mathrm{C}$ (Fig. 3), l'ordre local s'étend progressivement. A $250{ }^{\circ} \mathrm{C}$, le pic des $5 \mathrm{e}$ voisins dans la direction [10] à $12,5 \AA$ et celui des $4 \mathrm{e}$ voisins dans la direction [11] à $17,3 \AA$ apparaissent. Les oscillations de la fonction $p(r)$ ne sont plus négligeables à $20 \AA$ et se produisent au-delà : la distance de cohérence s'accroît dans la direction [002].

L'accroissement de l'étendue des rubans et celui de la taille des domaines associés au réarrangement des plans hexagonaux entraînent une augmentation de la conductivité. En effet, au-delà de $150^{\circ} \mathrm{C}$, les pics des $2^{\mathrm{e}}$ et $5^{\mathrm{e}}$ voisins de la fonction $p(r)$ et correspondant aux premières distances entre atomes de deux couches superposées s'amorcent respectivement en avant des pics à $4,30 \AA$ et $6,55 \AA$. Ils s'affirment au cours de recuits à température supérieure en même temps qu'ils migrent vers les faibles valeurs de $r$ pour occuper une position voisine de celle qui caractérise la structure hexagonale compacte, soit à $250^{\circ} \mathrm{C}: 3,70 \AA(3,54 \AA$ structure $\mathrm{HC})$ et $5,65 \AA$ (5,59 structure $\mathrm{HC})$.

En conclusion, le recuit de l'alliage maintenu dans le domaine 1 (non cristallisé) provoque une croissance des domaines, une orientation et un réarrangement des couches hexagonales, phénomènes qui le font tendre vers une structure hexagonale compacte dont la conductivité est nécessairement supérieure à celle de la substance initiale.

Ainsi le spectromètre de masse, l'analyse thermique différentielle et les diagrammes de diffraction des électrons et des rayons $\mathrm{X}$ permettent d'interpréter la variation irréversible de la conductivité électrique des lames minces de nickel phosphore du domaine 1, c'est-à-dire très imparfaitement cristallisées. 


\section{Bibliographie}

[1] Flechon (J.), Thèse Nancy, 1960.

[2] Machizaud (F.), Thèse 3e-cycle Nancy, 1965.

[3] Damask (A. C.) et Dienes (G. J.), Point Defects in Metal. Gordon and Breach, 1963, p. 148.

[4] DiXmier (J.), Thèse Orsay, 1969.

[5] Suhrmann (R.), Chemical Abstracts, 1956, 50-4, 11741-e.

[6] Mostovetch (N.), C. R. Acad. Sci., Paris, 1949, 228, 1702.

[7] Goldenstein (A. W.), J. Electrochem. Soc., 1957, 104, 104.

[8] Randin (J. P.), J. Electrochem. Soc., 1967, 114, 442.

[9] FleChON (J.), B. S. C. F., 1961, 1591.
[10] Turnbull (D.), J. Chem. Phys., 1968, 48, 2560.

[11] Flechon (J.) et Viard (M.), J. Phys. Appl., 1970, 5, 765.

[12] Maeda (H.), J. Appl. phys. Japan, 1969, 8, 978.

[13] Albert (P. A.) et Kovac (Z.), Phys. Rev., 1967, 38.

[14] Warnecke (R. J.), Ann. de Radioélectricité, 1957, $12,49$.

[15] Flechon (J.) et Machizaud (F.), Congrès international sur les couches minces, Cannes 1970, p. 519.

[16] Burke (J.), La cinétique des changements de phase dans les métaux, 1968, p. 7.

[17] Averbach (B. L.), Strong (S. L.) et Kaplov, J. phys. chem. Solids, 1964, 25, 1195. 